\title{
Over-Expression of Ephrin-A5 in Mice Results in Decreasing the Size of Progenitor Pool through Inducing Apoptosis
}

\author{
Hyuna Noh, and Soochul Park*
}

Eph receptors and their ligands, ephrins, mediate cell-tocell contacts in a specific brain region and their bidirectional signaling is implicated in the regulation of apoptosis during early brain development. In this report, we used the alpha( $\alpha)$-Cre transgenic line to induce ephrin$A 5$ over-expression in the distal region of the neural retina. Using this double transgenic embryo, we show that the over-expression of ephrin-A5 was responsible for inducing massive apoptosis in both the nasal and temporal retinas. In addition, the number of differentiated retinal neurons with the exception of the bipolar neuron was significantly reduced, whereas the laminar organization of the mature retina remained intact. Consistent with this finding, an analysis of the mature retina revealed that the size of the whole retina- particularly the nasal and temporal regions-is markedly reduced. These results strongly suggest that the level of ephrin-A5 expression plays a role in the regulation of the size of the retinal progenitor pool in the neural retina.

\section{INTRODUCTION}

Apoptosis is a key developmental process that is found frequently during early neural development in areas such as the vertebrate neural retina (Valenciano et al., 2009). Apoptosis in the developing retina has been classified into the following three phases: morphogenetic apoptosis, early neural cell apoptosis, and neurotrophic apoptosis. Morphogenetic apoptosis indicates the cell deaths that are observed during early morphogenetic eye events such as optic-vesicle evagination, optic cup invagination, and optic fissure closure (Morcillo et al., 2006; Ozeki et al., 2000). Early neural cell apoptosis represents the cell death that occurs in the neuronal progenitors or early born neurons of the developing neural retina (Cepko et al., 1996). Neurotrophic cell death refers to the cell death of differentiated

Department of Biological Science, Sookmyung Women's University, Seoul 140-742, Korea

*Correspondence: scpark@sookmyung.ac.kr

Received 15 September, 2015; revised 11 November, 2015; accepted 16 November, 2015; published online 15 December, 2015

Keywords: apoptotsis, ephrin-A5, retinal progenitor neurons that is mainly due to the inappropriate support of neurotrophic factors (Pequignot et al., 2003; Pollock et al., 2003). Whereas neurotrophic apoptosis has been intensively investigated, the underlying molecular mechanism of the early developmental phases of apoptosis remains poorly understood, despite the consideration of these phases as a key process of early eye morphogenesis or the regulation of the size of the progenitor pool.

Eph and ephrin signaling have been implicated in the inducement of apoptotic cell death in neuroepithelial cells during early brain development (Park, 2013). For example, it has been shown that the ectopic expression of ephrin-A5 in the EphA7-expressing neuroepithelial cells of the forebrain was responsible for a very effective inducement of massive cell death and the subsequent disorganization of brain development (Depaepe et al., 2005). Accordingly, researchers found that the brain size of an EphA7-deficient mouse embryo is larger due to a lower incidence of apoptotic cell death. We also showed that the dorsal midline of the dien- and mesencephalon is a region where EphA7 is co-expressed with ephrin-A5, and that the up-regulation of either ephrin- or Ephgene expression plays a causative role in the triggering of the pro-apoptotic signaling pathway (Kim et al., 2013; Park et al., 2013). These studies indicate that the regulation of ephrin- or Eph-gene expression may change cell-to-cell contact-based extrinsic signaling to trigger the pro-apoptotic pathway, thereby controlling the size of the neuronal progenitor pool or the remodeling of brain tissue.

In this study, we investigated whether the over-expression of ephrin-A5 can induce apoptotic cell death in retinal progenitors during neural-retina development. The evidences presented in this report strongly suggest that the level of ephrin-A5 has a key role in the determination of the size of the retinal progenitor pool due to its regulation of cell survival or death.

\section{MATERIALS AND METHODS}

\section{Mice}

The ephrin-A5 BAC transgenic mice expressing GFP have been previously described (Yoo et al., 2011). $\alpha$-cre transgenic mice, in which the Cre recombinase is controlled by a fragment of the Pax6 promoter were provided by Dr. Jinwoo Kim (KAIST) and have also been described previously (Marquardt et al., 2001). Gt(ROSA)26Sortm1(CAG-taulacZ)Bene (010633) mouse lines were purchased from The Jackson Laboratory. 
$\boldsymbol{A}$

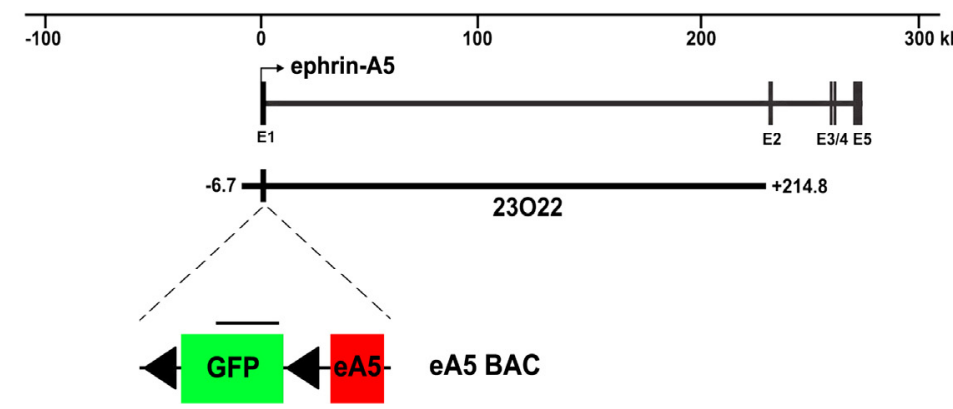

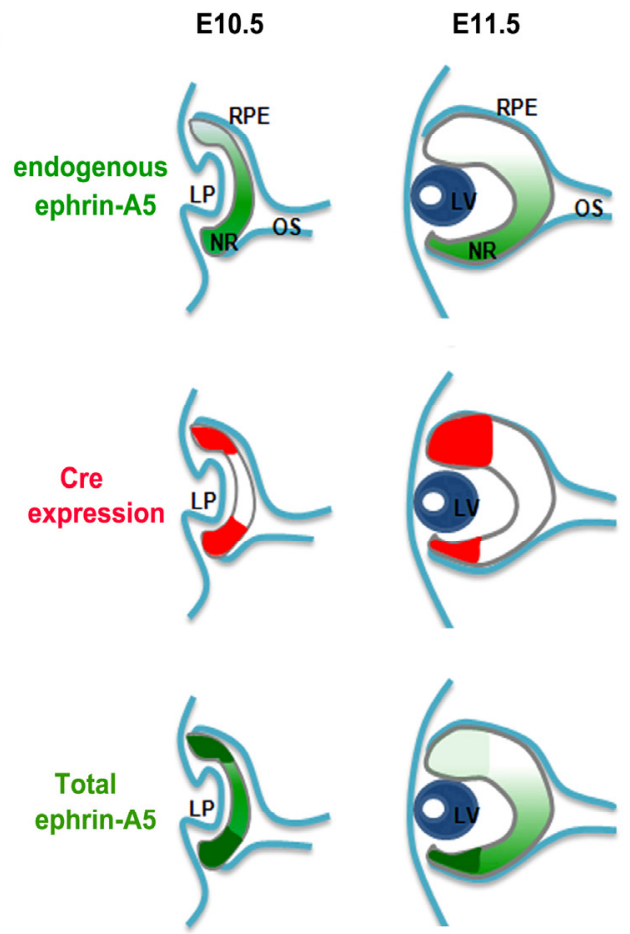

B

C

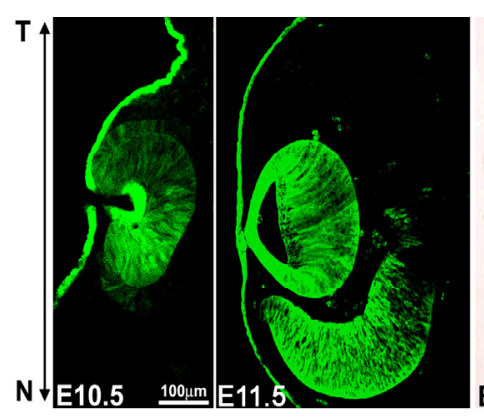

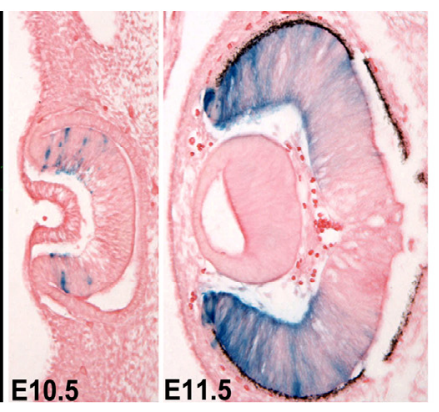

D

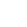

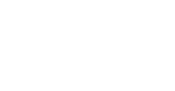

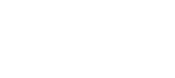

Fig. 1. Expression of ephrin-A5 by distal retina-specific Cre (A) Schematic map of a targeting vector for inserting Flag-tagged human ephrin-A5 cDNA into the ephrin-A5 BAC clone (RP23-23O22). (B) Horizontal sections showing GFP expression by ephrin-A5 BAC at different developmental stages. T (temporal); N (nasal). (C) Rosa-floxed STOP-LacZ mice were crossed with $\alpha$-Cre Tg mice to generate double Tg at the indicated developmental stages. Whole embryos were stained with X-gal and then subjected to sectioning along the horizontal plane. (D) Schematic diagram showing the predicted expression level of ephrin-A5 in the neural retina.

All of the mice were generated and maintained in accordance with the institutional guidelines approved by the Sookmyung Women's University Animal Care and Use Committee.

Immunohistochemistry, X-gal staining, and TUNEL assay For the IHC using antibodies were performed as described (Shim et al., 2007). Briefly, cryosections were washed in PBS. Then, the sections were immersed in blocking buffer $(10 \%$ horse serum, $0.3 \%$ Triton X-100 in PBS) for $1 \mathrm{~h}$ at room temperature, incubated with the primary antibody overnight at $4^{\circ} \mathrm{C}$, and lastly washed three times in PBS for 5 min. Subsequently, the slides were incubated with a suitable secondary antibody for $2 \mathrm{~h}$ at room temperature before they were washed in PBS. For X-gal staining, the embryos to be stained were dissected in PBS, fixed in $0.2 \%$ glutaraldehyde, and then washed and stained as described previously (Noh et al., 2014). TUNEL assays were performed according to the manufacturer's instructions (In Situ Cell Death Detection Kit, TMR red; Roche Diagnostics Corp., USA).

\section{Antibodies}

Immunostaining was performed by using polyclonal antibodies against cleaved caspase-3 (Cell Signaling), Brn-3 (Santa Cruz Biotechnology), PKC $\alpha$ (Santa Cruz Biotechnology), monoclonal antibodies against GFP (MBL), Flag (Sigma-Aldrich), calbindin (Sigma-Aldrich), and Syntaxin (Sigma-Aldrich).

\section{RESULTS}

Over-expression of ephrin-A5 results in inducement of apoptosis of retinal progenitors

To investigate the pro-apoptotic role of ephrin-A5 in the developing neural retina, we used a BAC transgenic strategy based on the Cre-dependent expression of ephrin-A5; this BAC clone (designated "eA5 BAC") contains a floxed GFP-STOP, followed by FLAG-tagged human ephrin-A5 cDNA (Fig. 1A). The eA5 BAC transgenic $(\mathrm{Tg})$ embryos revealed that GFP expression is relatively low in the optic cup at E10.5, but begins to increase mainly in the nasal retina at E11.5; this embryo does not express the FLAG-tagged human ephrin-A5 protein because of the stop signals between the GFP and ephrin-A5 cDNA (Fig. 1B). To induce the retina-specific expression of ephrin-A5, we used a $\alpha$-Cre $\mathrm{Tg}$ line; the enhancer-driven Cre activity was detected from as early as E10.5 and was confined exclusively to the distal retina of the Rosa26-LacZ reporter embryo (Fig. $1 \mathrm{C})$. Based on the expression pattern of both Cre and GFP, we predicted that the ephrin-A5 in the eA5- $\alpha$-Cre double Tg embryos would be over-expressed in both the nasal and temporal retinas at $E 10.5$, but that it would mainly occur in the nasal retina from $\mathrm{E} 11.5$ (Fig. 1D).

A TUNEL (terminal nucleotidyl transferase-mediated dUTP nick end labeling) staining analysis revealed that the Tg embryos carrying eA5 BAC (control embryos) barely contained apop- 


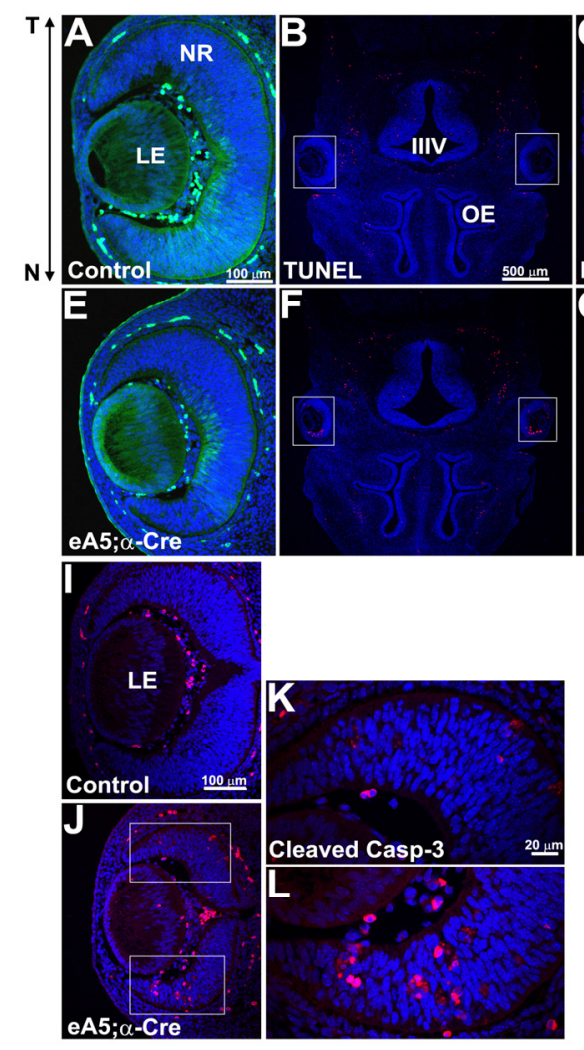

Fig. 2. Over-expression of ephrin-A5 in the neural retina promotes retinal cell apoptosis. (A, E) GFP expression in the horizontal sections of E13.5 embryos. The eA5 Tg embryo (control) shows a strong GFP expression in the nasal retina whereas the double Tg embryo does not. (B, F) TUNEL staining of horizontal sections from E13.5 littermate embryos. OE (olfactory epithelium). Boxed areas in $(B)$ and $(F)$ are enlarged in (C-D) and $(G-H)$. (I, J) IHC staining of horizontal sections using anticleaved caspase- 3 antibody. Boxed areas in (J) are enlarged in (K) and (L). (M) The data in (C) and $(G)$ were quantified. Data represent the mean \pm S.E. (eA5 Tg, 3; eA5T; $\alpha$-Cre Tg, 5). * $P$ and $P^{\star *}<$ 0.001 eA5̦; $\alpha$-Cre $\mathrm{Tg}$ versus eA5 $\mathrm{Tg}$, unpaired $t$ test. totic cells in the neural retina at E13.5 (Figs. 2A-2D). Importantly, the GFP expression in the eA5- $\alpha$-Cre double Tg embryo was no longer present in the nasal retina, and a significant number of cells (at least a 20 -fold increase) was concomitantly apoptotic in the same region that the GFP signal was removed (Figs. $2 \mathrm{E}-2 \mathrm{H}$, and $2 \mathrm{M}$ ). Apoptotic cell death in the temporal retina also increased by approximately 3-fold (Figs. $2 \mathrm{E}-2 \mathrm{H}$, and $2 \mathrm{M}$ ) and this is likely due to the over-expression of ephrin-A5 in this region at E10.5 (Figs. 1B-1D); furthermore, cleaved caspase-3 antibody staining revealed that the Cre-dependent ectopic expression of ephrin-A5 induced a caspase- 3 signaling cascade that is consistent with the TUNEL-staining analysis (Figs. 2l-2L). In addition, FLAG-tagged ephrin-A5 is ectopically expressed in the eA5- $\alpha$-Cre double $\mathrm{Tg}$ embryo and these ephrinA5expressing cells undergo apoptotic cell death, indicating that the ectopic ephrin-A5 induces apoptotic cell death in an autonomous manner (Supplementary Fig. 1). Taken together, our results demonstrate that the ephrin-A5 expression level is critically involved in the inducement of the pro-apoptotic signaling of retinal progenitors in the developing retina.

\section{Ephrin-A5 over-expression decreases the population size of retinal progenitors without disruption of laminar organization}

We next examined how the apoptotic cell death of retinal progenitors affected the postnatal retina of eA5- $\alpha$-Cre double Tg embryos. These mice were viable, fertile, and had normal life spans, and the only exception is that their eyes were morphologically smaller (microphthalmia phenotype) (Figs. 3A and 3D). The whole flat mount of the mature retina at postnatal $(P)$ day 21 revealed that the overall retina size of the double $\mathrm{Tg}$ mice is smaller than that of the control mice (Figs. 3B and 3E). Likewise, the optic nerve of the double $\mathrm{Tg}$ mice is much thinner, suggesting that the optic fibers from the retina are reduced (Figs. $3 \mathrm{C}$ and $3 \mathrm{~F}$ ). To test this hypothesis, triple $\mathrm{Tg}$ mice with eA5 BAC, tau-LacZ in the Rosa locus, and $\alpha$-Cre were generated (Figs. 3J-3L). Unlike the control mice, the triple Tg mice displayed a significant reduction in the number of LacZ-positive retinal ganglion cells (RGCs) in both the nasal and temporal retinas (Figs. 3G and 3J); furthermore, the number of LacZpositive axons was also reduced along the optic nerve (Figs. $3 \mathrm{H}$ and $3 \mathrm{~K})$, and their termination zones in the lateral geniculate nucleus (LGN), pretectum, and superior colliculus (SC) were also significantly reduced (Figs. $3 \mathrm{I}$ and $3 \mathrm{~L}$ ). Interestingly, the size of the SC from the triple Tg mice is smaller, whereas the intermediate region of the SC occupied by the unlabeled RGC axons (with no ephrin-A5 expression) is proportionately larger than that of the triple Tg mice (Figs. $3 \mathrm{I}$ and $3 \mathrm{~L}$ ). Consistent with these results, an immunofluorescent staining analysis for which the anti-Brn3 antibody was used revealed that the number of RGCs in the nasal retina of the double Tg mice decreased by approximately two-fold when compared with the control littermates (Figs. 3M-3T).

We further investigated whether the laminar structure of the nasal retina is disorganized in the double Tg mutants (Fig. 4). For this study, we performed immunofluorescent staining analyses using various retinal-neuron markers on the nasal retina where the GFP signal was eliminated by $\alpha$-Cre expression. As was expected, the numbers of horizontal, amacrine, and photoreceptor cells were significantly reduced in the double Tg mutants compared with those of the control mice (Figs. $4 \mathrm{~A}-4 \mathrm{H}, 4 \mathrm{M}$, $4 \mathrm{~N}$, and $4 \mathrm{O}$ ). In contrast, the number of bipolar cells was not significantly altered between the double $\mathrm{Tg}$ mutants and the control mice (Figs. 4I-4L); however, we observed that the overall laminar organization of the retinal neurons was not significantly disrupted in the double Tg mutants. Taken together, our 




Fig. 3. Over-expression of ephrin-A5 reduces retinal size. (A, D) Lateral view of right eyes from control and double $\mathrm{Tg}$ at P21. The whole flatmount retina from the mice in $(A)$ and $(D)$ is shown in $(B)$ and $(E)$, respectively. $D$ (dorsal); $V$ (ventral). (C, F) The entire retinofugal tissue containing the optic nerve $(\mathrm{ON})$, optic chiasm $(\mathrm{OC})$, and optic tract (OT) were dissected out of the mice in (A) and (D). (G-H and J-K) Experiments were performed as described in (A-F), except that the LacZ-positive RGCs and axons were visualized using whole mount $X$-gal staining. Note that the tau-LacZ targeted to the Rosa 26 (Ro) locus is expressed when the floxed stop signal is eliminated by Cre. $(I, L)$ The diencephalon and midbrain were dissected out of the mice in $(G)$ and $(J)$ and whole mount $\mathrm{X}$-gal staining was performed to show the topographic map from the LacZ-positive RGCs to the superior colliculus (SC). LGN (lateral geniculate nucleus); IC (inferior colliculus). (M, Q) Horizontal sections from the indicated mice at $\mathrm{P} 21$ were subjected to IHC staining using anti-Brn3 antibody to reveal the distribution of the retinal ganglion cells in the retina. Note that GFP expression was eliminated in the nasal retina of the double $\mathrm{Tg}$. Boxed area in $(\mathrm{M})$ and $(\mathrm{Q})$ is enlarged in (N-O) and (R-S), respectively. Boxed area in $(\mathrm{O})$ and $(\mathrm{S})$ is further enlarged in $(\mathrm{P})$ and $(\mathrm{T})$. findings demonstrate that the over-expression of ephrin-A5 results in a decrease of the population size of the retinal progenitors through the inducement of apoptotic cell death.

\section{DISCUSSION}

In this study, we show that the expression level of ephrin-A5 in retinal progenitors could be a critical factor in the modulation of the progenitor pool size during the development of the neural retina. The over-expression of ephrin-A5 was responsible for the inducement of massive apoptotic cell death among retinal progenitors, thereby decreasing the size of the adult retina and the numbers of retinal neurons with the exception of the bipolar neuron. An important issue to address is the underlying mechanism by which ephrin-A5 triggers pro-apoptotic signaling in the retinal progenitors during early eye development. Previous studies indicate that Eph-mediated signaling is capable of inducing apoptosis in neuroepithelial cells upon the engagement of ephrin ligands. However, we found that Eph receptors are not expressed in the nasal retina where apoptotic cells are predominantly detected due to the over-expression of ephrinA5; therefore, it is likely that a forward signaling from the ephrinA5 receptors to the Eph receptors is not involved in this apoptotic mechanism. Alternatively, some reports revealed that ephrin-A5 is involved in reverse signaling through an interaction with receptor tyrosine kinases (RTKs) such as RET (Klein and Kania, 2014); in this respect, it is conceivable that ephrin-A5 may interact with certain RTKs in the developing neural retina and that this interaction may be important for the regulation of the survival or differentiation of the retinal progenitors. For example, FGF receptors are highly expressed in proliferating retinal progenitors, and a severe dorsal-ventral patterning de- fect of the neural retina was observed in mouse mutants that were deficient in both FGFR1 and 2 (Lupo et al., 2005). A plausible hypothesis is that cross-talk between FGF RTK and ephrin-A5 may be an important factor for retinal cell differentiation. The temporal regulation of endogenous ephrin-A5 expression may block the rapid cell cycle of retinal progenitors and allow cells to differentiate into certain retinal neurons. Importantly, we observed that the ephrin-A5 expression was up-regulated from E11.5 when some of the retinal progenitors began to differentiate into neurons. The over-expression of ephrin-A5 may be more effective in interfering with the cell proliferation signaling of FGF RTK, thereby decreasing the cell survivability with a concomitant increase of pro-apoptotic signaling. Consistent with this hypothesis, we observed that the over-expression of ephrin-A5 in the developing neural retina is very effective at inhibiting cell proliferation among the retinal progenitors at E13.5 (data not shown). It would be especially interesting to investigate whether the retinal progenitors are more proliferative in the nasal retina of an ephrin-A5-null mutant embryo, although the functional redundancy among the ephrin genes could be an obstacle in the resolution of this hypothesis. Nevertheless, the findings that are presented in this report raise the interesting possibility that ephrin-A5 plays a role in the regulation of neurogenesis or the apoptosis of retinal progenitors during the development of the neural retina.

Note: Supplementary information is available on the Molecules and Cells website (www.molcells.org).

\section{ACKNOWLEDGMENTS}

This study was supported by Sookmyung Women's University Research Grants (1-1403-0075). 

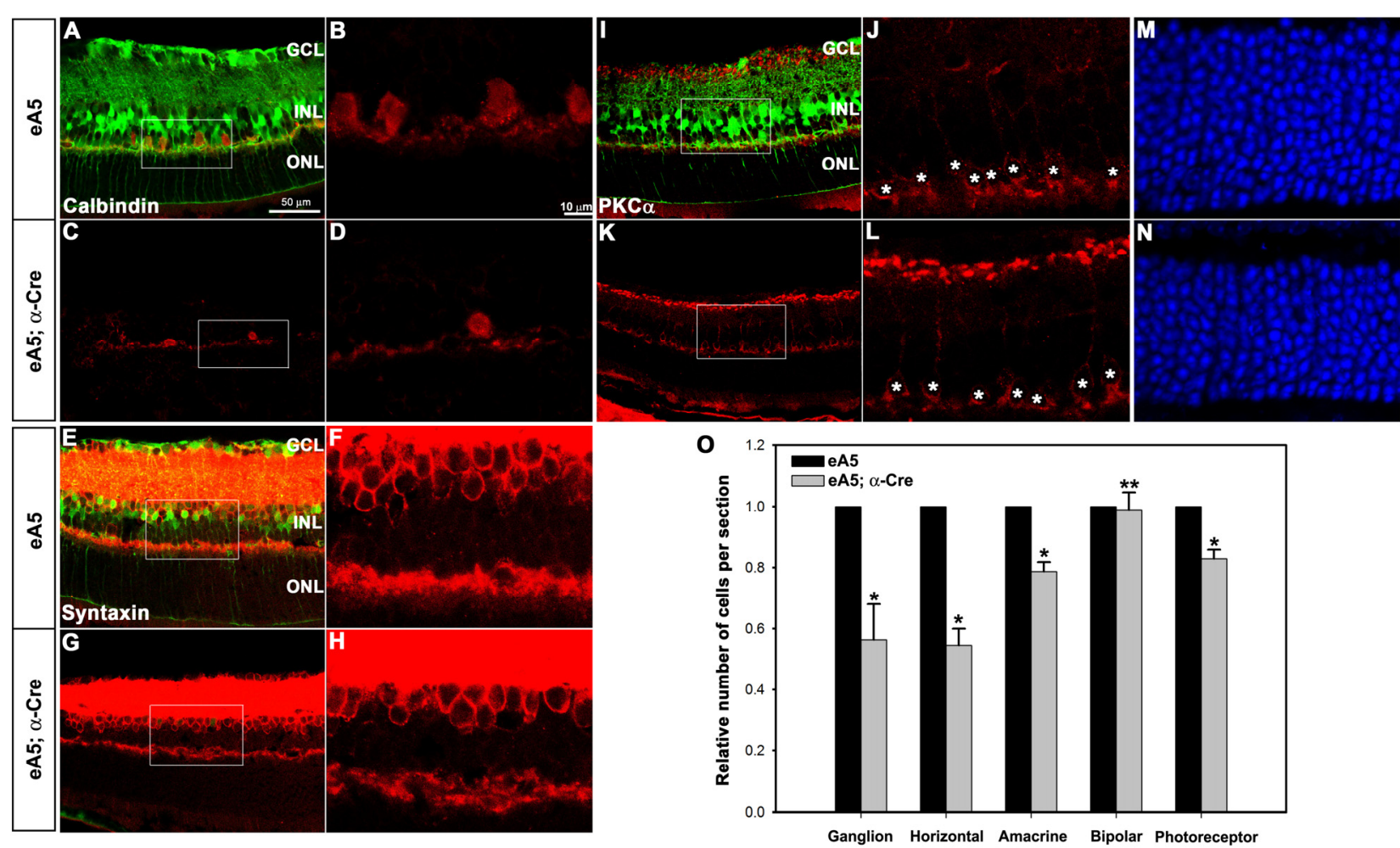

Fig. 4. Over-expression of ephrin-A5 results in a reduction of the retinal size without affecting the overall laminar organization. Horizontal sections were prepared from the indicated mice at P21. (A-N) IHC-staining analyses were performed using a variety of retinal neuron markers on the nasal retina where the GFP signal was eliminated by $\alpha$-Cre expression. Calbindin, horizontal cell marker; syntaxin, amacrine cell marker; $\mathrm{PKC} \alpha$, bipolar cell marker; DAPI staining was used to count the photoreceptors in the outer nuclear layer. white asterisk, bipolar cells. (O) The number of retinal cells was quantified. The number of retinal cells in each section of the control embryos was set to 1.0 to normalize the cells in the corresponding region of the double Tg embryos. Data represent the mean \pm S.E. (control embryo, 4; double $\mathrm{Tg}, 4$ ). ${ }^{*} P<0.003 ;{ }^{* *} P<0.1$

\section{REFERENCES}

Cepko, C.L., Austin, C.P., Yang, X., Alexiades, M., and Ezzeddine, D. (1996). Cell fate determination in the vertebrate retina. Proc. Natl. Acad. Sci. USA 93, 589-595.

Depaepe, V., Suarez-Gonzalez, N., Dufour, A., Passante, L., Gorski, J.A., Jones, K.R., Ledent, C., and Vanderhaeghen, P. (2005). Ephrin signalling controls brain size by regulating apoptosis of neural progenitors. Nature 435, 1244-1250.

Kim, Y., Park, E., Noh, H., and Park, S. (2013). Expression of EphA8-Fc in transgenic mouse embryos induces apoptosis of neural epithelial cells during brain development. Dev. Neurobiol. 73, 702-712.

Klein, R., and Kania, A. (2014). Ephrin signalling in the developing nervous system. Curr. Opin. Neurobiol. 27, 16-24.N

Lupo, G., Liu, Y., Qiu, R., Chandraratna, R.A., Barsacchi, G., He, R.Q., and Harris, W.A. (2005). Dorsoventral patterning of the Xenopus eye: a collaboration of Retinoid, Hedgehog and FGF receptor signaling. Development 132, 1737-1748.

Marquardt, T., Ashery-Padan, R., Andrejewski, N., Scardigli, R., Guillemot, F., and Gruss, P. (2001). Pax6 is required for the multipotent state of retinal progenitor cells. Cell 105, 43-55.

Morcillo, J., Martinez-Morales, J.R., Trousse, F., Fermin, Y., Sowden, J.C., and Bovolenta, P. (2006). Proper patterning of the optic fissure requires the sequential activity of BMP7 and SHH. Development 133, 3179-3190.

Noh, H., Park, E., and Park, S. (2014). In vivo expression of ephrinA5-Fc in mice results in cephalic neural crest agenesis and craniofacial abnormalities. Mol. Cells 37, 59-65.

Ozeki, H., Ogura, Y., Hirabayashi, Y., and Shimada, S. (2000).
Apoptosis is associated with formation and persistence of the embryonic fissure. Curr. Eye Res. 20, 367-372.

Park, S. (2013). Brain-region specific apoptosis triggered by eph/ephrin signaling. Exp. Neurobiol. 22, 143-148.

Park, E., Kim, Y., Noh, H., Lee, H., Yoo, S., and Park, S. (2013). EphA/ephrin-A signaling is critically involved in region-specific apoptosis during early brain development. Cell Death Differ. 20, 169-180.

Pequignot, M.O., Provost, A.C., Salle, S., Taupin, P., Sainton, K.M., Marchant, D., Martinou, J.C., Ameisen, J.C., Jais, J.P., and Abitbol, M. (2003). Major role of BAX in apoptosis during retinal development and in establishment of a functional postnatal retina. Dev. Dyn. 228, 231-238

Pollock, G.S., Robichon, R., Boyd, K.A., Kerkel, K.A., Kramer, M., Lyles, J., Ambalavanar, R., Khan, A., Kaplan, D.R., Williams, R.W., et al. (2003). TrkB receptor signaling regulates developmental death dynamics, but not final number, of retinal ganglion cells. J. Neurosci. 23, 10137-10145.

Shim, S., Kim, Y., Shin, J., Kim, J., and Park, S. (2007). Regulation of EphA8 gene expression by TALE homeobox transcription factors during development of the mesencephalon. Mol. Cell. Biol. 27, 1614-1630.

Valenciano, A.I., Boya, P., and de la Rosa, E.J. (2009). Early neural cell death: numbers and cues from the developing neuroretina. Int. J. Dev. Biol. 53, 1515-1528.

Yoo, S., Kim, Y., Noh, H., Lee, H., Park, E., and Park, S. (2011). Endocytosis of EphA receptors is essential for the proper development of the retinocollicular topographic map. EMBO J. 30 , 1593-1607. 